\title{
A Distributional Representation of Gamma Function with Generalized Complex Domian
}

\author{
Asifa Tassaddiq*, Rabia Safdar, Tahira Kanwal \\ Department of Mathematics, Government College University, Faisalabad, Pakistan \\ Email: *asifashabbir@gmail.com, rabia.safdar1109@gmail.com, kanwal.syed@live.com
}

How to cite this paper: Tassaddiq, A. Safdar, R. and Kanwal, T. (2017) A Distributional Representation of Gamma Function with Generalized Complex Domian. Advances in Pure Mathematics, 7, 441-449. https://doi.org/10.4236/apm.2017.78029

Received: June 19, 2017

Accepted: August 21, 2017

Published: August 24, 2017

Copyright $\odot 2017$ by authors and Scientific Research Publishing Inc. This work is licensed under the Creative Commons Attribution International License (CC BY 4.0).

http://creativecommons.org/licenses/by/4.0/

\section{(c) (i) Open Access}

\begin{abstract}
In this paper, we present a new representation of gamma function as a series of complex delta functions. We establish the convergence of this representation in the sense of distributions. It turns out that the gamma function can be defined over a space of complex test functions of slow growth denoted by $\mathcal{Z}$. Some properties of gamma function are discussed by using the properties of delta function.
\end{abstract}

\section{Keywords}

Gamma Function, Distributions or Generalized Functions, Fourier Transform, Dirac Delta Function, Space of Complex Test Functions

\section{Introduction}

The problem of giving $s$ ! a useful meaning when $s$ is any complex number was solved by Euler (1707-1783), who defined what is now called the gamma function,

$$
\Gamma(s):=\int_{0}^{\infty} \mathrm{e}^{-t} t^{s-1} \mathrm{~d} t \quad(s=\sigma+i \tau, \sigma=\mathfrak{R}(s)>0) .
$$

He extended the domain of gamma function from natural numbers, $\mathbb{N}$, to complex numbers $\mathbb{C}$. This is an extensively studied classical function and can be considered as a basic special function. It can be written as a sum of two incomplete gamma functions

$$
\Gamma(s):=\int_{0}^{1} \mathrm{e}^{-t} t^{s-1} \mathrm{~d} t+\int_{1}^{\infty} \mathrm{e}^{-t} t^{s-1} \mathrm{~d} t=\gamma(s, x)+\Gamma(s, x) .
$$

The function $\Gamma(s)$ itself, and its incomplete versions $\gamma(s, x)$ and $\Gamma(s, x)$, are known to play important role in the study of the analytic solutions of a variety of problems in diverse areas of science and engineering (see, for example, [1] [2] and [3]; see also the recent papers [4] [5] [6] and [7]). By using the 
Fourier transform representation, a distributional representation of the gamma function

$$
\Gamma(\sigma+i \tau)=2 \pi \sum_{m=0}^{\infty} \frac{(-1)^{m}}{m !} \delta(\tau-i(\sigma+m)) \quad(\sigma>0)
$$

was obtained in [8]. This led to new integral identities involving the gamma function.

At first glance, it seemed to give useful results but the "distributional representation" presented was not mathematically well defined there. As such, we could not be entirely sure of the validity of any new results presented in [8]. The consistency of the results obtained by classical means would be reliable. However, if we have to check each new result, the new representation would become useless. The problems with the distributional representation were that the delta functional along the imaginary axis had not been defined in [8], nor was a clear definition of the space of test functions provided.

Similarly, the results presented were generalized in [9]

$\Gamma_{b}(\sigma+i \tau)=2 \pi \sum_{n=0}^{\infty} \sum_{m=0}^{\infty} \frac{(-1)^{n+m} b^{n}}{m ! n !} \delta(\tau-i(\sigma+m-n)) \quad(\Re(b)>0 ; b=0, \sigma>0)$.

Again some new formulas were derived, which were also checked by using classical means but the representation was not mathematically well defined. Such type of representations are also obtained for other special functions, see for example [10] and [11].

In this paper, we present a new representation of gamma function and establish the convergence of this representation in the sense of distributions. By doing so it turns out that the domain of gamma function can be extended from complex numbers $\mathbb{C}$ to complex functions $\mathcal{Z}$. Some properties of gamma function are discussed by using the properties of delta function.

The plan of this paper is as follows. After giving a necessary and brief introduction to distributions and test functions in Section 2, we discuss the distributional representation of gamma function in Section 3. The convergence of the series along with its properties are also discussed here. Some properties of gamma function w.r.t Fourier transformation are discussed in Section 4.

\section{Distributions and Test Functions}

The space of all continuous linear functionals acting on the space of test functions with compact support and its dual are denoted by $\mathcal{D}$ and $\mathcal{D}^{\prime}$ respectively. Distributions can be generated by using the following method. Let $f(t)$ be a locally integrable function then corresponding to it, one can define a distribution $f$ through the convergent integral

$$
\langle f, \phi\rangle=\langle f(t), \phi(t)\rangle:=\int_{-\infty}^{+\infty} f(t) \phi(t) \mathrm{d} t \quad \forall \phi \in \mathcal{D} .
$$

It is easy to show that it is a continuous linear functional by using the definition of $\phi$ and the details are omitted here, [12]. Distributions that can be 
generated in this way (from locally integrable functions) are called the regular distributions. The study of distributions is important because they not only contain representations of locally integrable functions but also include many other entities that are not regular distributions. Therefore many operations like limits, integration and differentiation, which were originally defined for functions, can be extended to these new entities. All distributions that are not regular are called singular distributions, for example, the delta function is a singular distribution.

The Fourier transform of an arbitrary distribution in $\mathcal{D}^{\prime}$ is not, in general, a distribution but is instead another kind of continuous linear functional which is defined over a new space of test functions. Such a functional is called an ultradistribution, for example delta function of complex argument is an ultradistribution ([12], Section (7.7)). Some properties of delta function include

$$
\begin{gathered}
\langle\delta(s-c), \phi(s)\rangle=\phi(s) \quad(\forall \phi \in \mathcal{Z}, c \in \mathbb{C}), \\
\delta(-s)=\delta(s) ; \delta(\alpha s)=\frac{1}{|\alpha|} \delta(s) \quad(\alpha \neq 0)
\end{gathered}
$$

and

$$
\delta(a-s) \delta(s-b)=\delta(a-b) \text { for } a>|s|>b .
$$

Therefore, we first discuss the space of test functions on which such distributions act. The space of test functions denoted by $\mathcal{Z}$ consists of all those entire functions whose Fourier transforms are the elements of $\mathcal{D}$, ([12], Section (7.6)). Since $\phi$ is an entire function, it cannot be zero on any interval $a<t<b$ except when it is zero everywhere. Thus the spaces $\mathcal{D}$ and $\mathcal{Z}$ do not intersect except in the identically zero testing function. The corresponding dual to the testing function space $\mathcal{Z}$ is $\mathcal{Z}^{\prime}$ whose elements are the Fourier transforms of the elements of $\mathcal{D}^{\prime}$. Neither $\mathcal{D}$ nor $\mathcal{D}^{\prime}$ are subspaces of $\mathcal{Z}$ or $\mathcal{Z}^{\prime}$ and vice virsa. However, the following inclusion holds

$$
\mathcal{Z} \subset \mathcal{S} \subset \mathcal{S}^{\prime} \subset \mathcal{Z}^{\prime}
$$

and

$$
\mathcal{D} \subset \mathcal{S} \subset \mathcal{S}^{\prime} \subset \mathcal{D}^{\prime} .
$$

Here $\mathcal{S}$ is the space of testing functions of rapid descent and $\mathcal{S}^{\prime}$ is the space of distributions of slow growth, which is also called the space of tempered distributions. An important example of ultradistributions is ([12], p. 204, Equation (7))

$$
\mathcal{F}\left[\mathrm{e}^{\alpha t} ; \omega\right]=2 \pi \sum_{n=0}^{\infty} \frac{(i \alpha)^{n}}{n !} \delta^{n}(\omega)=2 \pi \delta(\omega+i \alpha),
$$

where $\alpha$ is a complex number. Hence, delta function of complex argument is defined on the space of test functions $\mathcal{Z}$.

A sequence $\left\{f_{\mu}\right\}_{\mu=1}^{\infty}$ is said to convergent in $\mathcal{Z}^{\prime}$ if every $f_{\mu}$ is in $\mathcal{Z}^{\prime}$ and if, for each $\phi$ in $\mathcal{Z}$, the sequence $\left\{\left\langle f_{\mu}, \phi\right\rangle\right\}_{\mu=1}^{\infty}$ converges in the ordinary 
sense of convergence of numbers. As $\phi$ traverses $\mathcal{Z}$, the limits of $\left\{\left\langle f_{\mu}, \phi\right\rangle\right\}_{\mu=1}^{\infty}$ define a functional $f$ on $\mathcal{Z}$, and $f$ is said to be the limit of $\left\{f_{\mu}\right\}_{\mu=1}^{\infty}$. The dual space $\mathcal{Z}^{\prime}$ is closed under convergence. As usual, an infinite series is said to converge in $\mathcal{Z}^{\prime}$ if the sequence of its partial sums converges in $\mathcal{Z}^{\prime}$. For further discussion of the spaces, $\mathcal{Z}$ and $\mathcal{Z}^{\prime}$, see ([12], Chapter 2) and ([12], Chapter 3).

\section{Distributional Representation of the Gamma Function and Some Properties}

Theorem 1. Gamma function has a series representation

$$
\Gamma(s)=2 \pi \sum_{n=0}^{\infty} \frac{(-1)^{n}}{n !} \delta(s+n) .
$$

Proof. Consider Equation (1.2)

$$
\Gamma(\sigma+i \tau)=2 \pi \sum_{n=0}^{\infty} \frac{(-1)^{n}}{n !} \delta(\tau-i(\sigma+n)) .
$$

Now, by making use of the basic property of delta function given in Equation (2.3) and taking $\sigma+i \tau=s$, we obtain

$$
\delta(\tau-i(\sigma+n))=\delta\left[\frac{1}{i}\left(i \tau-i^{2}(\sigma+n)\right)\right]=|i| \delta(\sigma+i \tau+n)=\delta(s+n),
$$

which leads to (3.1).

Theorem 2. Prove that (3.1) converges over the space of complex test functions denoted by $\mathcal{Z}$.

Proof. The series can act over test functions as

$$
\langle\Gamma(s), \phi(s)\rangle=2 \pi \sum_{n=0}^{\infty} \frac{(-1)^{n}}{n !} \phi(-n) .
$$

We can note that

$$
\sum_{m=0}^{\infty} \frac{(-1)^{n}}{n !}=\frac{1}{\mathrm{e}}
$$

and $\phi(-n)$ is slowly increasing sequence of test functions. Therefore, the series (3.4) is convergent by using famous Abel convergent test or by ([13], Proposition 1, p. 46)

Remark 1. Note that $\phi \in \mathcal{Z}$ implies that the series is convergent. Conversely if the series is convergent then $\phi$ may or may not belong to $\mathcal{Z}$, therefore the condition is necessary and not sufficient.

Remark 2. Note that (3.1) generalizes the domain of (1.2) from real $\tau$ to complex $s$ and then to $\phi(s)$.

Remark 3. Any sequence of complex delta functions $\{\delta(s+n)\}_{n=0}^{\infty}$, when multiplied and divided by $\frac{2 \pi}{\mathrm{e}}$ gives us $\frac{\mathrm{e}}{2 \pi}$ times gamma function 


$$
\frac{2 \pi \sum_{n=0}^{\infty} \frac{(-1)^{n}}{n !}}{2 \pi \sum_{n=0}^{\infty} \frac{(-1)^{n}}{n !}} \delta(s+n)=\frac{\mathrm{e}}{2 \pi} \Gamma(s) .
$$

Theorem 3. Prove that gamma function is a continuous linear functional over $\mathcal{Z}$.

Proof. If $\phi_{1}(s)$ and $\phi_{2}(s)$ are test functions in $\mathcal{Z}$ and $\alpha$ and $\beta$ are two complex numbers, then

$$
\left\langle\Gamma(s), \alpha \phi_{1}(s)+\beta \phi_{2}(s)\right\rangle=\left\langle 2 \pi \sum_{n=0}^{\infty} \frac{(-1)^{n}}{n !} \delta(s+n), \alpha \phi_{1}(s)+\beta \phi_{2}(s)\right\rangle .
$$

Since complex delta is linear over $\mathcal{Z}$, this implies that gamma function is also linear over $\mathcal{Z}$. It gives us

$$
\left\langle\Gamma(s), \alpha \phi_{1}(s)+\beta \phi_{2}(s)\right\rangle=\alpha\left\langle\Gamma(s), \phi_{1}(s)\right\rangle+\beta\left\langle\Gamma(s), \phi_{2}(s)\right\rangle .
$$

If $\Gamma(s)$ is known to be linear, definition of continuity will be wane to as "the numerical sequence $\left\{\left\langle\Gamma(s), \phi_{v}(s)\right\rangle\right\}_{v=1}^{\infty}$ converges to zero, whenever the sequence converges to zero." Let $\left\{\phi_{\mu}\right\}_{\mu=1}^{\infty}$ be a sequence of test functions in $\mathcal{Z}$ converges to zero. Then by the continuity of delta functional, the sequence $\left\{\left\langle\delta(s+n), \phi_{\mu}\right\rangle\right\}_{\mu=1}^{\infty}$ converges to zero, which implies that

$$
\left\{\left\langle\Gamma(s), \phi_{\mu}\right\rangle\right\}_{\mu=1}^{\infty}=2 \pi \sum_{n=0}^{\infty} \frac{(-1)^{n}}{n !}\left\{\left\langle\delta(s+n), \phi_{\mu}\right\rangle\right\}_{\mu=1}^{\infty}
$$

converges to zero. For linear functionals continuity at any point is equivalent to continuity at every point. It proves that gamma function (3.1) is a continuous linear functional over $\mathcal{Z}$

Theorem 4. Prove that following properties hold for gamma function as a distribution.

1) Addition

$$
\left\langle\Gamma(s), \phi_{1}(s)+\phi_{2}(s)\right\rangle=\left\langle\Gamma(s), \phi_{1}(s)\right\rangle+\left\langle\Gamma(s), \phi_{2}(s)\right\rangle,
$$

2) Multiplication

$$
\langle\alpha \Gamma(s), \phi(s)\rangle=\langle\Gamma(s), \alpha \phi(s)\rangle,
$$

$\alpha$ be any complex or real number.

3) Shifting or Translation

$$
\langle\Gamma(s-\tau), \phi(s)\rangle=\langle\Gamma(s), \phi(s+\tau)\rangle .
$$

4) Multiplication of independent variable with a constant (positive or negative)

$$
\langle\Gamma(a s), \phi(s)\rangle=\left\langle\Gamma(s), \frac{1}{a} \phi\left(\frac{s}{a}\right)\right\rangle .
$$

5) Shifting including multiplication with independent variable 


$$
\langle\Gamma(a s-\tau), \phi(s)\rangle=\left\langle\Gamma(s), \frac{1}{a} \phi\left(\frac{s}{a}+\tau\right)\right\rangle .
$$

6) The product of gamma function with a regular distribution $\psi(s)$ is defined over $\mathcal{Z}$.

7) The product $\psi(s) \Gamma(s)$ is also continuous linear functional over $\mathcal{Z}$.

Proof. Results (1-7) can easily be proved by using the properties of complex delta functions.

Theorem 5. Prove that distributional derivatives of gamma function also exist.

Proof. By using $\left\langle\delta^{(1)}(s), \phi(s)\right\rangle=-\phi^{(1)}(0),([12]$, p.14, (12)), first derivative of (3.1) is

$$
\left\langle\Gamma^{(1)}(s), \phi(s)\right\rangle=\sum_{n=0}^{\infty} \frac{(-1)^{n}}{n !}(-1)^{1} \phi^{1}(-n),
$$

which is convergent as discussed earlier. Similarly for $k^{\text {th }}$ order implies that

$$
\left\langle\Gamma^{k}(s), \phi(s)\right\rangle=\sum_{n=0}^{\infty} \frac{(-1)^{n}}{n !}(-1)^{k} \phi^{k}(-n) .
$$

Theorem 6. The recurrence relation for gamma function as a distribution holds true $\Gamma(s+1)=s \Gamma(s)$ iff $\phi(s-1)=s \phi(s)$ where $\phi \in \mathcal{Z}$.

Proof. By using the Equation (3.10) and fixing $\alpha$ as a complex number" $s$ " and using Equation (3.11), one can obtain the required result.

Theorem 7. The following result for the product of the gamma function holds true

$$
\Gamma(a-s) \Gamma(s-b)=(2 \pi / \mathrm{e})^{2} \delta(a-b), \forall \phi(s) \in \mathcal{Z} .
$$

Proof. Consider

$$
\begin{aligned}
& \langle\Gamma(a-s) \Gamma(s-b), \phi(s)\rangle \\
& =\left(2 \pi \sum_{n=0}^{\infty} \frac{(-1)^{n}}{n !}\right)^{2}\langle\delta(a-(s+n)) \delta((s+n)-b), \phi(s)\rangle
\end{aligned}
$$

By making use of (2.4), we get

$$
\begin{gathered}
\langle\Gamma(a-s) \Gamma(s-b), \phi(s)\rangle=\left(2 \pi \sum_{n=0}^{\infty} \frac{(-1)^{n}}{n !}\right)^{2}\langle\delta(a-b), \phi(s)\rangle \\
\langle\Gamma(a-s) \Gamma(s-b), \phi(s)\rangle=(2 \pi / \mathrm{e})^{2}\langle\delta(a-b), \phi(s)\rangle .
\end{gathered}
$$

Hence proved.

\section{Properties of Distributional Representation of Gamma Function w.r.t Fourier Transformation}

Before stating the properties, we write the necessary notations used for this section. Here

$$
\mathcal{F}[\Gamma(s)]=\tilde{\Gamma}(s) ; \mathcal{F}[\phi](s)=\tilde{\phi}(s) .
$$




$$
\begin{aligned}
\langle\tilde{\Gamma}(s), \phi(s)\rangle & =2 \pi \sum_{n=0}^{\infty} \frac{(-1)^{n}}{n !}\langle\tilde{\delta}(s+n), \phi(s)\rangle \\
& =2 \pi \sum_{n=0}^{\infty} \frac{(-1)^{n}}{n !}\langle\delta(s+n), \tilde{\phi}(s)\rangle=\langle\Gamma(s), \tilde{\phi}(s)\rangle .
\end{aligned}
$$

By using ([12], p. 184) gives

$$
\langle\tilde{\Gamma}(s), \tilde{\phi}(s)\rangle=2 \pi\langle\Gamma(t), \phi(-t)\rangle=2 \pi\langle\Gamma(t), \hat{\phi}(t)\rangle,
$$

where $\hat{\phi}$ denotes the transpose of test function $\phi$.

$$
\langle\bar{\Gamma}(s), \tilde{\phi}(s)\rangle=2 \pi\langle\tilde{\Gamma}(s), \overline{\tilde{\phi}(s)}\rangle=2 \pi\langle\tilde{\Gamma}(s), \overline{\phi(-t)}\rangle=2 \pi\langle\Gamma(t), \check{\phi}(t)\rangle,
$$

here we define $\overline{\phi(-t)}=\breve{\phi}(t)$

$$
\langle\tilde{\Gamma}(s), \overline{\tilde{\phi}(s)}\rangle=2 \pi\langle\tilde{\Gamma}(s), \overline{\phi(-t)}\rangle=2 \pi\langle\Gamma(t), \check{\phi}(t)\rangle
$$

Again, from parseval's identity

$$
\langle\overline{\tilde{\Gamma}(s)}, \overline{\tilde{\phi}(s)}\rangle=2 \pi\langle\tilde{\Gamma}(s), \overline{\overline{\tilde{\phi}(s)}}\rangle=2 \pi\langle\tilde{\Gamma}(s), \tilde{\phi}(s)\rangle
$$

By applying double Fourier transform

$$
\langle\tilde{\Gamma}(s), \phi(s)\rangle=2 \pi\langle\tilde{\Gamma}(s), \tilde{\phi}(s)\rangle=2 \pi\langle\Gamma(t), \phi(-t)\rangle
$$

i.e., double Fourier transform of a distribution is again a distribution. There will be a change of sign in range as one can see ([6], p. 189, problem(1)).

Theorem 8. By using the differentiation and integration rules of distribution prove that

$$
\tilde{\Gamma}^{k}(s)=\left[(-i t)^{k} \Gamma(t)\right] .
$$

Proof. For $\phi$ is in $\mathcal{Z}$,

$$
\begin{gathered}
\left\langle\tilde{\Gamma}^{(1)}(s), \phi(s)\right\rangle=\left\langle\tilde{\Gamma}(s), \phi^{(1)}(s)\right\rangle \\
\left\langle\tilde{\Gamma}^{(1)}(s), \phi(s)\right\rangle=\left\langle\Gamma(s), \tilde{\phi}^{(1)}(s)\right\rangle \\
\left\langle\tilde{\Gamma}^{(1)}(s), \phi(s)\right\rangle=\left\langle\Gamma(s),(-i t) \phi(s) \mathrm{e}^{-i s t}\right\rangle \\
\left\langle\tilde{\Gamma}^{(1)}(s), \phi(s)\right\rangle=\langle(-i t) \Gamma(s), \tilde{\phi}(s)\rangle \\
\left\langle\tilde{\Gamma}^{(1)}(s), \phi(s)\right\rangle=\langle(-i t) \tilde{\Gamma}(s), \phi(s)\rangle .
\end{gathered}
$$

On the same way differentiation w.r.t $s$ upto $k$ times, we get

$$
\left\langle\tilde{\Gamma}^{k}(s), \phi(s)\right\rangle=\left\langle(-i t)^{k} \tilde{\Gamma}(s), \phi(s)\right\rangle .
$$

It gives

$$
\tilde{\Gamma}^{k}(s)=(-i t)^{k} \tilde{\Gamma}(s)
$$

or

$$
\tilde{\Gamma}^{k}(s)=(-i t)^{k} \Gamma(t) .
$$


Theorem 9. If $\Gamma(s)$ is in $\mathcal{Z}^{\prime}$ then its Fourier $\tilde{\Gamma}(s)$ is also in $\mathcal{Z}^{\prime}$.

Proof. Since $\mathcal{Z}^{\prime}$ is closed under Fourier transformation therefore result follows.

Theorem 10. If $\Gamma(s)$ is a distribution of complex space $\mathcal{Z}$ then its Fourier transformation $\tilde{\Gamma}(t)$ is an infinitely smooth distribution.

Proof. By following the lines of above theorem which shows that derivative of Fourier transform of $\Gamma(s)$ exist upto $k^{\text {th }}$ order in distributional sense. So $\tilde{\Gamma}(t)$ is a distribution that is infinitely smooth.

\section{Concluding Remarks}

One normally thinks of a function as defined by a series or an integral of some variable or in terms of those functions that we regard as "elementary". However, the function needs to be regarded as an entity in itself, which is represented by a series or an integral. Only in this sense can we continue the function beyond its original domain of definition. This is essential for applying the function beyond dealing with the problem it was originally defined to deal with. This consideration becomes especially important when discussing the theory of (special) functions. There is more than one representation for all special functions, for example, the series representation, the asymptotic representation, the integral representation, etc. Further there can be more than one integral or series representation, which help to define the function in different regions. For example the integral representation (1.1) of the gamma function is defined in the positive half of the complex plane. It leads to another representation, which is defined in the whole complex plane except at negative integers. One can also use a different representation to express the function in terms of simpler functions. It will help to know the behavior of the original function at certain points of its domain. One representation can give simpler proofs of some known properties as compared to other. Here, in our present investigation, we have obtained a new representation of gamma function, which defines it over a space of complex entire test functions of slow growth denoted by $\mathcal{Z}$. Some properties of gamma function are also discussed over this space. It is hoped that various new properties and applications of gamma function can also be defined using this generalized domain. It will extend the use of gamma function in distribution theory and other fields of Engineering and Science.

\section{Acknowledgements}

The authors are thankful to the reviewers for their useful comments.

\section{References}

[1] Chaudhry, M.A. and Zubair, S.M. (2001) On a Class of Incomplete Gamma Functions with Applications. Chapman and Hall (CRC Press), Boca Raton. https://doi.org/10.1201/9781420036046

[2] Spanier, J. and Old-Ham, K.B. (1987) An Atlas of Functions, Hemisphere Publishing Corporation, New York. 
[3] Temme, N.M. (1996) Special Functions: An Introduction to Classical Functions of Mathematical Physics. John Wiley and Sons, New York, Chichester, Brisbane and Toronto. https://doi.org/10.1002/9781118032572

[4] Lin, S.D., Srivastava, H.M. and Yao, J.C. (2015) Some Classes of Generating Relations Associated with a Family of the Generalized Gauss Type Hypergeometric Functions. Applied Mathematics \& Information Sciences, 9, 1731-1738.

[5] Rehman, A., Mubeen, S., Sadiq, N. and Shaheen, F. (2014) Some Inequalities Involving k-Gamma and k-Beta Functions with Applications. Journal of Inequalities and Applications, 224, 1-16.

[6] Srivastava, R., Agarwal, R. and Jain, S. (2017) A Family of the Incomplete Hypergeometric Functions and Associated Integral Transform and Fractional Derivative Formulas. Filomat, 31, 125-140. https://doi.org/10.2298/FIL1701125S

[7] Srivastava, H.M., Chaudhry, M.A. and Agarwal, R.P. (2012) The Incomplete Pochhammer Symbols and Their Applications to Hypergeometric and Related Functions. Integral Transforms and Special Functions, 23, 659-683.

https://doi.org/10.1080/10652469.2011.623350

[8] Chaudhry, M.A. and Qadir, A. (2004) Fourier Transform and Distributional Representation of Gamma Function Leading to Some New Identities. International Journal of Mathematics and Mathematical Sciences, 37, 2091-2096.

https://doi.org/10.1155/S016117120430743X

[9] Tassaddiq, A. and Qadir, A. (2011) Fourier Transform and Distributional Representation of the Generalized Gamma Function with Some Applications. Applied Mathematics and Computation, 218, 1084-1088.

https://doi.org/10.1016/j.amc.2011.03.075

[10] Tassaddiq, A. (2011) Some Representations of the Extended Fermi-Dirac and BoseEinstein Functions with Applications. Ph.D. Dissertation, National University of Sciences and Technology Islamabad, Pakistan.

[11] Al-Lail, M.H. and Qadir, A. (2015) Fourier Transform Representation of the Generalized Hypergeometric Functions with Applications to the Confluent and Gauss Hypergeometric Functions. Applied Mathematics and Computation, 263, 392-397. https://doi.org/10.1016/j.amc.2015.04.083

[12] Zamanian, A.H. (1987) Distribution Theory and Transform Analysis. Dover Publications, New York.

[13] Richards, I. and Youn, H. (2007) Theory of Distributions: A Non Technical Introduction. Cambridge University Press, Cambridge. 
Submit or recommend next manuscript to SCIRP and we will provide best service for you:

Accepting pre-submission inquiries through Email, Facebook, LinkedIn, Twitter, etc. A wide selection of journals (inclusive of 9 subjects, more than 200 journals)

Providing 24-hour high-quality service

User-friendly online submission system

Fair and swift peer-review system

Efficient typesetting and proofreading procedure

Display of the result of downloads and visits, as well as the number of cited articles Maximum dissemination of your research work

Submit your manuscript at: http://papersubmission.scirp.org/

Or contact apm@scirp.org 